\title{
Stationary Distribution Convergence for Generalized Jackson Networks in Heavy Traffic*
}

\author{
Amarjit Budhiraja \\ Department of Statistics and Operations Research \\ University of North Carolina \\ Chapel Hill, NC 27599
}

\author{
Chihoon Lee \\ Department of Statistics \\ Colorado State University \\ Fort Collins, CO 80523
}

September 9, 2008

\begin{abstract}
In a recent paper [5] it was shown that under suitable conditions stationary distributions of the (scaled) queue lengths process for a generalized Jackson network converge to the stationary distribution of the associated reflected Brownian motion in the heavy traffic limit. The proof relied on certain exponential integrability assumptions on the primitives of the network. In this note we show that the above result holds under much weaker integrability conditions. We provide an alternative proof of this result assuming (in addition to natural heavy traffic and stability assumptions) only standard independence and square integrability conditions on the network primitives that are commonly used in heavy traffic analysis. Furthermore, under additional integrability conditions we establish convergence of moments of stationary distributions.
\end{abstract}

Keywords: invariant measures, generalized Jackson network, reflected Brownian motion, heavy traffic analysis.

AMS Subject Classifications: primary 60G35; secondary 60J25, 60K25, 93E15.

\section{Introduction}

In a recent paper [5], the authors have shown that under appropriate conditions the stationary distributions of suitably scaled queue length processes for generalized Jackson networks (GJN) converge to the stationary distribution of the associated reflected Brownian motion (RBM) in the heavy traffic limit. One of the key assumptions made in the analysis is that the inter-arrival and service times have finite moment generating functions (m.g.f.) in the neighborhood of origin (the precise assumption is a bit stronger and formulated in terms of residual service times and arrival times at time zero). The proof is based on strong approximation techniques and detailed uniform (in

\footnotetext{
${ }^{*}$ Research supported in part by the Army Research Office (Grant W911NF-0-1-0080).
} 
the scaling parameter) estimates on certain exponential moments of the state process. Finiteness of the m.g.f. of the primitive processes is a critical ingredient in these estimates. Indeed, it is suggested in [5] that for primitives with certain Pareto like distributions, steady state of RBM may be a poor approximation for the corresponding physical network. In this note we provide an elementary proof of the above result in [5] under much weaker integrability conditions. We make standard independence and second moment assumptions on inter-arrival and service times; see (A1) - (A4) in Section 2. These assumptions are typically used in heavy traffic analysis for invoking a functional central limit theorem [6]. In addition, similar to [5], we assume the heavy traffic condition (A5), a natural stability condition, (A6) and a nondegeneracy condition (A7).

Our proof is based on uniform stability estimates on a family of certain deterministic dynamical systems obtained from a fluid limit analysis of the underlying queueing networks. Using Lipschitz property of the associated Skorohod map, these estimates yield uniform (in the scaling parameter) moment bounds as in Theorem 3.3 for the scaled state processes of the GJN. We then use Lyapunov function methods of [10] and [4] developed in the study of time uniform moment bounds for Markov processes. By suitably adapting the proof of Proposition 5.3 of [4], we obtain, in Theorem 3.4, estimates on moments of the state process that are uniform in both time and the scaling parameter. From these estimates, moment bounds for the stationary distributions of the GJN, uniform in the scaling parameter, follow readily yielding tightness of the collection of these distributions. Our main result on convergence of invariant distributions is then immediate on combining this tightness property with the functional central limit result of [11]. We also show that by suitably strengthening moment conditions on the underlying renewal processes (see (A8.p) in Section 3) one can obtain convergence of moments of stationary distributions as well.

The paper is organized as follows. In Section 2.1.1 we recall the formulation of a GJN and introduce basic assumptions on the inter-arrival and service time distributions. A description of the dynamics of the queue length process in terms of a Skorohod map is presented in Section 2.1.2. Diffusion scaling and appropriate heavy traffic conditions are introduced in Section 2.2.1. We also recall in this section the basic heavy traffic limit result of [6]. In Section 2.2.2 our main stability assumption is introduced and the well known results of [4] and [7] on existence of steady state distributions for GJN and RBM, respectively, are recalled. Section 3 presents our main result (Theorem 3.1) that establishes weak convergence of the stationary queue length distributions for the diffusion scaled GJN networks to the unique stationary distribution of the RBM.

The following notation will be used. For a metric space $X$, let $\mathcal{B}(X)$ be the Borel $\sigma$-field on $X$ and $\mathbb{I}(X)$ the collection of all probability measures on $X$. The set of positive integers is denoted by $\mathbb{N}$, the set of real numbers by $\mathbb{R}$ and non-negative real numbers by $\mathbb{R}_{+}$. Let $\mathbb{R}^{d}$ be the $d$ dimensional Euclidean space and for $x \in \mathbb{R}^{d}$ the $L_{1}$ norm of $x$, i.e., $\sum_{i=1}^{d}\left|x_{i}\right|$, will be denoted by $|x|$. For a given matrix $M$ denote by $M^{\prime}$ its transpose and by $M^{i}$ the $i^{\text {th }}$ row of $M$. Let $\mathbb{I}=\mathbb{I}_{K \times K}$ denote the identity matrix for given $K$. When clear from the context, we will omit the subscript. For $a, b \in \mathbb{R}$, let $a \vee b \doteq \max \{a, b\}, a \wedge b \doteq \min \{a, b\}$ and $a^{+} \doteq \max \{0, a\}$. The convergence in distribution of random variables (with values in some Polish space) $\Phi_{n}$ to $\Phi$ will be denoted as $\Phi_{n} \Rightarrow \Phi$. With an abuse of notation weak convergence of probability measures (on some Polish space) $\nu_{n}$ to $\nu$ will also be denoted as $\nu_{n} \Rightarrow \nu$. For a Polish space $Y$ let $D([0, \infty), Y)$ denote the class of right continuous functions with left limits defined from $[0, \infty)$ to $Y$ with the usual Skorohod topology. For $m \in \mathbb{P}(Y)$ and an $m$-integrable function $f$, we will denote $\int_{Y} f(x) d m(x)$ by $\langle m, f\rangle$. 
Inequalities for vectors are interpreted componentwise.

\section{Problem Formulation}

Let $(\Omega, \mathcal{F}, \mathbb{P})$ be a probability space. Unless specified otherwise, all the random variables considered in this work are assumed to be defined on this probability space.

\subsection{Generalized Jackson network}

Network structure. We start by describing a network with $K$ service stations, denoting the $i^{\text {th }}$ station by $P_{i}, i \in \mathbb{K} \doteq\{1, \ldots, K\}$. We assume that each station has an infinite capacity buffer. We consider a single class network, that is, all customers at a station are homogeneous in terms of service requirement and routing decision. Arrivals of jobs can be from outside the system and/or from internal routing. Upon completion of service at station $P_{i}$ a customer is routed to other service stations (or exits the system) according to a probabilistic routing matrix $\mathcal{P}$. At every station the jobs are assumed to be processed by First-In-First-Out discipline. We assume that the network is open, that is, any customer entering the network eventually leaves it. (See below (2.2) for a precise formulation.) This network was considered by Jackson in [8] with exponential inter-arrival/service time distributions and is referred to as Jackson network in this Markovian setting. We allow general inter-arrival and service time distributions. Hereafter, this single class network will be referred to as a generalized Jackson network (GJN).

\subsubsection{Stochastic primitives and assumptions}

For $k \in \mathbb{N}$, let $\eta_{i}(k), \Delta_{i}(k)$ denote the $k^{\text {th }}$ inter-arrival time and $k^{\text {th }}$ service time, respectively, at station $P_{i}$, since time 0 . (We only consider exogenous arrivals here.) We assume:

(A1) For $\ell, i \in \mathbb{K},\left\{\eta_{\ell}(k): k \geq 1\right\},\left\{\Delta_{i}(k): k \geq 1\right\}$ are i.i.d. sequences with values in $[0, \infty]$.

Additional independence conditions on these sequences of random variables will be specified below in this section (see (A3)). A station $P_{j}$ is said to have non-null exogenous arrivals if $\mathbb{P}\left[\eta_{j}(1)<\right.$ $\infty]>0$. Let $\mathcal{E}$ denote the set of indices of stations with non-null exogenous arrivals. Whenever external arrivals are under discussion, we consider only the non-null exogenous arrivals. Our second assumption on the network is the following:

(A2) For $\ell \in \mathcal{E}$ and $i \in \mathbb{K}, \mathbb{E}\left[\eta_{\ell}(1)^{2}\right] \doteq \gamma_{l}^{\eta}<\infty$ and $\mathbb{E}\left[\Delta_{i}(1)^{2}\right] \doteq \gamma_{i}^{\Delta}<\infty$.

Denote for $i \in \mathcal{E}, \alpha_{i} \doteq 1 / \mathbb{E}\left[\eta_{i}(1)\right]$ the external arrival rate into station $P_{i}$ and for $i \notin \mathcal{E}$, we set $\alpha_{i}, \gamma_{i}^{\eta}$ equal to 0 . Then $\alpha=\left(\alpha_{1}, \ldots, \alpha_{K}\right)^{\prime}$ is the vector of external arrival rates. Let $m_{i} \doteq \mathbb{E}\left[\Delta_{i}(1)\right]$ denote the mean service time at station $P_{i}$ and $\mu_{i} \doteq 1 / m_{i}$ the service rate at $P_{i}$. Set $\mu=\left(\mu_{1}, \ldots, \mu_{K}\right)^{\prime}$ and $M$ to be the diagonal matrix with $m_{1}, \ldots, m_{K}$ as diagonal entries. We assume $\mu_{i}$ and $\alpha_{\ell}$ are finite 
for $i \in \mathbb{K}$ and $\ell \in \mathcal{E}$. Denote by $\gamma^{\eta}$ the vector $\left(\gamma_{1}^{\eta}, \cdots \gamma_{K}^{\eta}\right)^{\prime}$. The vector $\gamma^{\Delta}$ is defined similarly. Let $\gamma=\left(\gamma^{\eta}, \gamma^{\Delta}\right)$.

For $i \in \mathbb{K}$, let $U_{i, 0}$ and $V_{i, 0}$ be random variables representing the residual inter-arrival time and service time at time 0 , at $P_{i}$. Here we adopt the convention $U_{i, 0}=\infty$ for $i \notin \mathcal{E}$. Requirements on independence of these random variables from other network primitives will be specified below (see (A3)). Define for $t \geq 0$,

$$
A_{i}(t) \doteq \max \left\{r \geq 1: \eta_{i}(0)+\eta_{i}(1)+\cdots+\eta_{i}(r-1) \leq t\right\}, \quad i \in \mathbb{K},
$$

where $\eta_{i}(0) \doteq U_{i, 0}$ and we follow the convention that max over an empty set is 0 . Thus $A_{i}(t)$ represents the total number of arrivals at $P_{i}$ by time $t$. Denote by $D_{j}(t)$ the total number of service completions at $P_{j}$ by time $t$ and let $D_{j i}(t)$ be the number of those jobs that are routed to $P_{i}$ immediately upon completion at station $P_{j}$. Denote by $Q_{i}(t)$ the queue length at time $t$, i.e., number of customers that are in queue or currently in service at $P_{i}$. Then, for $i \in \mathbb{K}$,

$$
Q_{i}(t)=Q_{i}(0)+A_{i}(t)-D_{i}(t)+\sum_{j=1}^{K} D_{j i}(t) .
$$

The routing decisions at each station are to be of Bernoulli type. More precisely, we consider a $K \times K$ sub-stochastic matrix $\mathcal{P}=\left(p_{j i}\right)_{j, i \in \mathbb{K}}$, where the entry $p_{j i}$ is the probability of the event that upon completion at $P_{j}$ the job is routed to station $P_{i}$. The spectral radius of the transition matrix $\mathcal{P}$ is assumed to be strictly less than unity, which ensures that all customers eventually leave the network. For $i \in \mathbb{K}$ and each $k \in \mathbb{N}$, let $\phi^{i}(k)$ be the routing (column) vector for the $k^{\text {th }}$ customer at station $P_{i}$ upon finishing service. Then $\phi^{i}(k)$ is a $K$-dimensional "Bernoulli random vector" with parameter $\left(\mathcal{P}^{i}\right)^{\prime}$, where $\mathcal{P}^{i}$ denotes the $i^{\text {th }}$ row of $\mathcal{P}$. By this we mean that, $\phi^{i}(k)=e_{j}$ with probability $p_{i j}$ and $\phi^{i}(k)=\mathbf{0}$ with probability $1-\sum_{j=1}^{K} p_{i j}$. Here $e_{j}$ is the $K$-dimensional $j^{\text {th }}$ coordinate vector. We assume

(A3) The random variables $\left\{\eta_{i}(k), \Delta_{i}(k), \phi^{i}(k): i \in \mathbb{K}, k \geq 1\right\}$ are independent. Also, this collection of random variables is independent of $\left\{U_{i, 0}, V_{i, 0}, Q_{i}(0): i \in \mathbb{K}\right\}$.

Define

$$
R^{i}(k) \doteq \sum_{l=1}^{k} \phi^{i}(l)
$$

which measures aggregated routing decisions up to $k^{\text {th }}$ service completion at station $P_{i}$. In particular, $R_{j}^{i}(k)$ will denote the $j^{\text {th }}$ component of $R^{i}(k)$, representing total number of routings from $P_{i}$ to $P_{j}$ among the first $k$ services completed.

Let $E_{i}(t)$ be the total number of service completions at the station $P_{i}$ in $t$ units of service time since time 0 . Note that $E_{i}$ in general will be different from $D_{i}$ due to service idleness. I.e.,

$$
E_{i}(t) \doteq \max \left\{r \geq 1: \Delta_{i}(0)+\Delta_{i}(1)+\cdots+\Delta_{i}(r-1) \leq t\right\},
$$

where $\Delta_{i}(0) \doteq V_{i, 0}$ and as before, max over an empty set is 0 . Also let $T_{i}(t)$ be the cumulative amount of service time that the station $P_{i}$ has spent on customers by time $t$. Let $I_{i}(t) \doteq t-T_{i}(t)$ 
denote the amount of time that the station $P_{i}$ has been idle by time $t$. We assume the network is non-idling, that is, a service station is idle only when there are no customers at that station requiring service. Then

$$
D_{j}(t)=E_{j}\left(T_{j}(t)\right), \quad D_{j i}(t)=R_{i}^{j}\left(E_{j}\left(T_{j}(t)\right)\right) .
$$

Henceforth, we will refer to $\alpha, \mu, \gamma$ and $\mathcal{P}$ as the parameters of the GJN. We define traffic intensity vector $\rho=\left(\rho_{1}, \ldots, \rho_{K}\right)^{\prime}$ of this GJN as $\rho_{i} \doteq\left(\left[\mathbb{I}-\mathcal{P}^{\prime}\right]^{-1} \alpha\right)_{i} / \mu_{i}, i \in \mathbb{K}$. Initial condition of the network is specified by random variables $\left(Q_{i}(0), U_{i, 0}, V_{i, 0}: i \in \mathbb{K}\right)$.

\subsubsection{System dynamics and Skorohod mapping}

The evolution of the state of the system satisfies the following equations: For $i \in \mathbb{K}$,

$$
\begin{aligned}
& Q_{i}(t)=Q_{i}(0)+A_{i}(t)-E_{i}\left(T_{i}(t)\right)+\sum_{j=1}^{K} R_{i}^{j}\left(E_{j}\left(T_{j}(t)\right)\right), \\
& \int_{0}^{\infty} Q_{i}(t) d I_{i}(t)=0 .
\end{aligned}
$$

We note that these processes satisfy,

$$
Q_{i}(t) \geq 0, \quad T_{i} \text { and } I_{i} \text { are non-decreasing and } T_{i}(0)=I_{i}(0)=0, \quad i \in \mathbb{K} .
$$

Equations (2.4), (2.5) and (2.6) describe the system dynamics. Next consider the "centered" process $\widetilde{Q} \doteq\left(\widetilde{Q}_{i}(t): t \geq 0, i \in \mathbb{K}\right)$, where

$$
\begin{aligned}
\widetilde{Q}_{i}(t) \doteq & Q_{i}(0)+\left(A_{i}(t)-\alpha_{i} t\right)-\left(E_{i}\left(T_{i}(t)\right)-\mu_{i} T_{i}(t)\right)+\sum_{j \in \mathbb{K}}\left(R_{i}^{j}\left(E_{j}\left(T_{j}(t)\right)\right)-p_{j i} E_{j}\left(T_{j}(t)\right)\right) \\
& +\sum_{j \in \mathbb{K}} p_{j i}\left(E_{j}\left(T_{j}(t)\right)-\mu_{j} T_{j}(t)\right)+\left(\alpha_{i}+\sum_{j \in \mathbb{K}} \mu_{j} p_{j i}-\mu_{i}\right) t .
\end{aligned}
$$

Denote $Y_{i} \doteq \mu_{i} I_{i}$. Set $Q \doteq\left(Q_{1}, \ldots, Q_{K}\right)^{\prime}$ and analogously define $T, I, E$, and $Y$. The dynamics in $(2.4)$ - (2.6) can now be represented in the following succinct vector forms:

$$
\begin{aligned}
& Q(t)=\widetilde{Q}(t)+\left[\mathbb{I}-\mathcal{P}^{\prime}\right] Y(t), \quad t \in \mathbb{R}_{+} \\
& \int_{0}^{\infty} Q_{i}(t) d Y_{i}(t)=0, \quad i \in \mathbb{K}, \\
& Q(t) \geq 0, \quad Y \text { is non-decreasing. }
\end{aligned}
$$

The above dynamics can equivalently be stated in terms of a Skorohod map as we describe below.

Definition 2.1. Let $\psi \in D\left([0, \infty), \mathbb{R}^{K}\right)$ be given with $\psi(0) \in S \doteq \mathbb{R}_{+}^{K}$. Then $(\phi, \eta) \in D\left([0, \infty), \mathbb{R}^{K}\right) \times$ $D\left([0, \infty), \mathbb{R}^{K}\right)$ solves the Skorohod problem for $\psi$ with respect to $S$ and $\left[\mathbb{I}-\mathcal{P}^{\prime}\right]$ if and only if the following hold: 
(i) $\phi(t)=\psi(t)+\left[\mathbb{I}-\mathcal{P}^{\prime}\right] \eta(t) \in S$, for all $t \geq 0$;

(ii) $\eta$ satisfies, for $i \in \mathbb{K}$, (a) $\eta_{i}(0)=0$, (b) $\eta_{i}$ is nondecreasing, and (c) $\eta_{i}$ can increase only when $\phi$ is on the $i^{\text {th }}$ face of $S$, that is, $\int_{0}^{\infty} 1_{\left\{\phi_{i}(s) \neq 0\right\}} d \eta_{i}(s)=0$.

Let $D_{S}\left([0, \infty), \mathbb{R}^{K}\right) \doteq\left\{\psi \in D\left([0, \infty), \mathbb{R}^{K}\right): \psi(0) \in S\right\}$. On the domain $D \subset D_{S}\left([0, \infty), \mathbb{R}^{K}\right)$ on which there is a unique solution to the Skorohod problem (SP) we define the Skorohod map (SM) $\Gamma$ as $\Gamma(\psi) \doteq \phi$, if $\left(\phi,\left[\mathbb{I}-\mathcal{P}^{\prime}\right]^{-1} \phi-\psi\right)$ is the unique solution of the SP posed by $\psi$. The following result (see [6]) gives the regularity of the Skorohod map defined by the data $\left(S,\left[\mathbb{I}-\mathcal{P}^{\prime}\right]\right)$.

Proposition 2.2. The Skorohod map is well defined on all of $D_{S}\left([0, \infty), \mathbb{R}^{K}\right)$, that is, $D=$ $D_{S}\left([0, \infty), \mathbb{R}^{K}\right)$, and the $S M$ is Lipschitz continuous in the following sense. There exists a constant $R \in(1, \infty)$ such that for all $\phi_{1}, \phi_{2} \in D_{S}\left([0, \infty), \mathbb{R}^{K}\right)$ :

$$
\sup _{0 \leq t<\infty}\left|\Gamma\left(\phi_{1}\right)(t)-\Gamma\left(\phi_{2}\right)(t)\right|<R \sup _{0 \leq t<\infty}\left|\phi_{1}(t)-\phi_{2}(t)\right| .
$$

Equivalent form of dynamics (2.8) - (2.10) in terms of the SM can now be written as follows:

$$
Q=\Gamma(\widetilde{Q}), \quad Q-\widetilde{Q}=\left[\mathbb{I}-\mathcal{P}^{\prime}\right] Y .
$$

\subsection{GJN in heavy traffic}

\subsubsection{Heavy traffic approximations}

Under appropriate heavy traffic conditions, the queue lengths of suitably scaled GJN can be approximated by a diffusion with constant coefficients, constrained to take values in $S=\mathbb{R}_{+}^{K}$. More precisely, consider a sequence of GJN networks indexed by $n \in \mathbb{N}$ with parameters $\left(\alpha^{n}, \mu^{n}, \mathcal{P}\right)$. Assumptions (A1) through (A3) are assumed to hold for each fixed $n$. Processes $Q^{n}, \widetilde{Q}^{n}, Y^{n}, M^{n}, T^{n}$ are defined in a manner analogous to Section 2.1.2. In particular, (2.8) - (2.10) and (2.12) hold with $(Q, \widetilde{Q}, Y)$ replaced by $\left(Q^{n}, \widetilde{Q}^{n}, Y^{n}\right)$. The following uniform integrability condition, which is standard in the study of central limit theorems for triangular arrays, will be used.

(A4) The family $\left\{\left(\eta_{l}^{n}(1)\right)^{2},\left(\Delta_{i}^{n}(1)\right)^{2}, l \in \mathcal{E}, i \in \mathbb{K}, n \in \mathbb{N}\right\}$ is uniformly integrable.

Finally, the heavy traffic assumption we make is as follows:

(A5) $\gamma^{n} \rightarrow \gamma, \quad \alpha^{n}=\alpha-\frac{\tilde{v}^{n}}{\sqrt{n}}, \quad \tilde{v}^{n} \rightarrow \tilde{v}, \quad \mu^{n}=\mu-\frac{\tilde{\beta}^{n}}{\sqrt{n}}, \quad \tilde{\beta}^{n} \rightarrow \tilde{\beta}, \quad\left[\mathbb{I}-\mathcal{P}^{\prime}\right]^{-1} \alpha=\mu$.

Here $\alpha, \mu, \tilde{v}^{n}, \tilde{\beta}^{n}, \tilde{v}, \tilde{\beta} \in \mathbb{R}^{K}$ and $\gamma \in \mathbb{R}^{K} \times \mathbb{R}^{K}$. Also $\mu^{n}, \mu$ are strictly positive and $\alpha^{n}, \alpha \geq 0\left(\alpha_{i}\right.$, $\alpha_{i}^{n}$ are strictly positive when $i \in \mathcal{E}$ and 0 when $\left.i \notin \mathcal{E}\right)$. Note that the traffic intensity vector $\rho^{n}$ of the $n^{\text {th }}$ GJN can be written as

$$
\rho_{i}^{n}=1-\frac{\left(\left[\mathbb{I}-\mathcal{P}^{\prime}\right]^{-1} \tilde{v}^{n}\right)_{i}-\tilde{\beta}_{i}^{n}}{\sqrt{n} \mu_{i}^{n}},
$$


and as $n \rightarrow \infty, \rho^{n} \rightarrow 1$.

To state the precise heavy traffic limit result, we begin with the following Markovian state description for GJN. Recall the initial residual times, $U_{i, 0}, V_{i, 0}$ introduced in Section 2.1.1. We denote the analogous quantities for the $n^{\text {th }}$ GJN by $U_{i, 0}^{n}$ and $V_{i, 0}^{n}$, respectively. For $t \geq 0$, and $i \in \mathbb{K}$, let $U_{i}^{n}(t)$ and $V_{i}^{n}(t)$ denote the remaining time, at instant $t$, before the next exogenous arrival and next service completion, respectively, at station $P_{i}$, for the $n^{\text {th }}$ GJN. They can be explicitly written as follows:

$$
U_{i}^{n}(t)=U_{i}^{n}(0)+\sum_{k=1}^{A_{i}^{n}(t)} \eta_{i}^{n}(k)-t, \quad V_{i}^{n}(t)=V_{i}^{n}(0)+\sum_{k=1}^{D_{i}^{n}(t)} \Delta_{i}^{n}(k)-T_{i}^{n}(t) .
$$

Define the state of the system at time $t$ at $P_{i}$ by

$$
X^{n}(t) \doteq\left(Q_{i}^{n}(t), U_{\ell}^{n}(t), V_{i}^{n}(t): i \in \mathbb{K}, \ell \in \mathcal{E}\right) .
$$

Let $X^{n}=\left(X^{n}(t): t \geq 0\right)$. Note that the process $\left(Q^{n}(t): t \geq 0\right)$ alone is not Markovian due to the residual inter-arrival/service times, but one can check that the augmented process $\left(X^{n}(t): t \geq 0\right)$ is indeed a strong Markov process with state space $\mathbb{X} \doteq \mathbb{R}_{+}^{K} \times \mathbb{R}_{+}^{|\mathcal{E}|} \times \mathbb{R}_{+}^{K}$. See [3] for details. Define the diffusion scaled processes:

$$
\widehat{X}^{n}(t) \doteq \frac{X^{n}(n t)}{\sqrt{n}}, \quad \widehat{W}^{n}(t) \doteq \frac{\widetilde{Q}^{n}(n t)}{\sqrt{n}}, \quad \widehat{I}^{n}(t) \doteq \frac{I^{n}(n t)}{\sqrt{n}} .
$$

We next recall the definition of a reflected Brownian motion.

Definition 2.3. Let $\left\{\mathcal{F}_{t}\right\}$ be a filtration on $(\Omega, \mathcal{F}, \mathbb{P})$. For $\mu_{0} \in \mathbb{P}\left(\mathbb{R}_{+}^{K}\right), b \in \mathbb{R}^{K}$ and a positive definite $K \times K$ matrix $\Sigma$, a reflected Brownian motion (RBM) associated with the data $(b, \Sigma)$ with initial distribution $\mu_{0}$, is a continuous $\left\{\mathcal{F}_{t}\right\}$-adapted $K$-dimensional process $Z$ such that

(i) $Z=\Gamma(Z(0)+B+b \iota), \mathbb{P}$-a.s. Here $\iota:[0, \infty) \rightarrow[0, \infty)$ is the identity map.

(ii) $B$ is a $K$-dimensional $\left\{\mathcal{F}_{t}\right\}$-Brownian motion with covariance $\Sigma$, mean $\mathbf{0}$ and $B(0)=\mathbf{0}$.

(iii) $Z(0)$ is distributed according to $\mu_{0}$.

We will write $\xi(t) \doteq Z(t)-Z(0)-B(t)-b t, t \geq 0$ and refer to $Z$ as a $(b, \Sigma)-R B M$.

It is well known that $Z$ is a strong Markov process. The following result follows upon slightly modifying the arguments in [11]. We remark that [11] considers a single collection of primitives (inter-arrival and service times) which is then suitably scaled for heavy traffic analysis. For the setting of a sequence of collections satisfying (A4), (A5) as considered here, the proofs are analogous to [11].

Recall that $\widehat{Q}^{n}(t) \doteq \frac{Q^{n}(n t)}{\sqrt{n}}$. 
Theorem 2.4. Assume that the sequence of measures $\mathbb{P} \circ\left[\widehat{X}^{n}(0)\right]^{-1}$ converges weakly to some $\nu \in \mathbb{P}(\mathbb{X})$. Then the process $\left(\widehat{Q}^{n}, \widehat{W}^{n}, M_{n}^{-1} \widehat{I}^{n}\right)$ converges in distribution in $D\left([0, \infty), \mathbb{R}^{K}\right)^{\otimes 3}$ to $(Z, B+b, \xi)$ as in Definition 2.3 with $\mu_{0}$ given as $\mu_{0}(A) \doteq \nu\left(A \times \mathbb{R}_{+}^{|\mathcal{E}|} \times \mathbb{R}_{+}^{K}\right)$, for $A \in \mathcal{B}\left(\mathbb{R}_{+}^{K}\right)$; $b=\left[\mathbb{I}-\mathcal{P}^{\prime}\right] \tilde{\beta}-\tilde{v}$; and some non-negative definite matrix $\Sigma$.

The matrix $\Sigma$ can be written explicitly in terms of the limit parameters $(\alpha, \mu, \gamma, \mathcal{P})$.

\subsubsection{Stability}

We now introduce our main stability condition on the sequence of GJN that will be used in this work.

(A6) There exists $\theta \in \mathbb{R}^{K}, \theta>0$, such that, for all $n \in \mathbb{N}$,

$$
M_{n}\left[\tilde{\beta}^{n}-\left[\mathbb{I}-\mathcal{P}^{\prime}\right]^{-1} \tilde{v}^{n}\right]<-\theta .
$$

Note that $(\mathrm{A} 6)$ is equivalent to the requirement that for some $\theta_{0} \in(0, \infty)$,

$$
\sup _{n} \max _{i \in \mathbb{K}} \sqrt{n}\left(\rho_{i}^{n}-1\right) \leq-\theta_{0}<0 .
$$

In particular we have that for each fixed $n, \max _{i} \rho_{i}^{n}<1$. This traffic intensity property implies the stability of GJN for each fixed $n$ as summarized in the following result from [12]. (See also [9] and [4].)

Theorem 2.5. Under assumptions of Section 2.2.1 and (A6), there exists a stationary probability distribution for the Markov process $\widehat{X}^{n}$.

We remark that, in general uniqueness of invariant measures for $\widehat{X}^{n}$ may not hold unless additional conditions are imposed. In what follows, if the initial condition of the Markov process $\widehat{X}^{n}$ is $x$ for some $x \in \mathbb{X}$, i.e., $\left(\widehat{Q}^{n}(0), \widehat{U}^{n}(0), \widehat{V}^{n}(0)\right) \equiv(q, u, v) \doteq x$, we will write the process as $\widehat{X}_{x}^{n}$.

Next we consider stability of RBM. We will impose the following nondegeneracy condition:

(A7) $\Sigma$ is positive definite.

The following theorem from [7] gives a necessary and sufficient condition for positive recurrence of a $(b, \Sigma)$-RBM.

Theorem 2.6. Under assumptions of Theorem 2.5 and (A7), the RBM with data $(b, \Sigma)$ has a unique stationary probability distribution $\pi$ if and only if $\left[\mathbb{I}-\mathcal{P}^{\prime}\right]^{-1} b<0$.

Note that (A6) in particular implies that $\tilde{\beta}-\left[\mathbb{I}-\mathcal{P}^{\prime}\right]^{-1} \tilde{v}<0$ and so from Theorem 2.6, under (A6), a $(b, \Sigma)$-RBM has a unique stationary probability distribution, where $b$ and $\Sigma$ are as in Theorem 2.4. Recall that we denote this probability measure on $\mathbb{R}_{+}^{K}$ by $\pi$. 


\section{Convergence of invariant measures}

All the assumptions of Section 2 will hold throughout this section and explicit reference to them in statement of our results will be omitted. The following additional condition will be used for proving convergence of moments of stationary distributions. Define $A_{i, 0}^{n}$ and $E_{i, 0}^{n}$ by (2.1), (2.3) with $U_{i, 0}^{n}$ and $V_{i, 0}^{n}$ there replaced by 0 . Let $p \in[2, \infty)$.

(A8.p) For some $c_{p} \in(0, \infty)$ :

(i) For all $i, j \in \mathbb{K}, n \geq 1$ and $t \geq 0$

$$
\mathbb{E} \sup _{0 \leq s \leq t}\left(\left|A_{i, 0}^{n}(s)-\alpha_{i}^{n} s\right|^{p}+\left|E_{i, 0}^{n}(s)-\mu_{i}^{n} s\right|^{p}+\left|R_{i}^{j}\left(E_{j, 0}^{n}(s)\right)-p_{j i} E_{j, 0}^{n}(s)\right|^{p}\right) \leq c_{p}\left(1+t^{p / 2}\right) .
$$

(ii) For all $i \in \mathcal{E}, j \in \mathbb{K}, n \geq 1 \mathbb{E}\left(\left|\Delta_{j}^{n}(1)\right|^{p}+\left|\eta_{i}^{n}(1)\right|^{p}\right) \leq c_{p}$.

From standard estimates for renewal processes (see e.g., [2] Lemma 3.5), we see that under assumptions of Section 2, (A8.p) holds for $p=2$. More generally, we refer the reader to [13] for sufficient conditions on the primitives of renewal processes under which (A8.p) is satisfied.

The following is the main result of this paper. Recall that from Theorem 2.5, for each $n \in \mathbb{I}$, the Markov process $\widehat{X}^{n}$ admits a stationary probability measure $\pi_{n}$. Denote by $\pi_{n}^{0}$ the marginal distribution of $\pi_{n}$ on the first coordinate of $\mathbb{X}$, i.e., $\pi_{n}^{0}(A)=\pi_{n}\left(A \times \mathbb{R}_{+}^{|\mathcal{E}|} \times \mathbb{R}_{+}^{K}\right), A \in \mathcal{B}\left(\mathbb{R}_{+}^{K}\right)$.

Theorem 3.1. The sequence $\left\{\pi_{n}^{0}\right\}$ of probability measures on $\left(\mathbb{R}_{+}^{K}, \mathcal{B}\left(\mathbb{R}_{+}^{K}\right)\right)$ converges weakly to the unique invariant probability measure, $\pi$, of the $(b, \Sigma)-R B M$, where $b$ and $\Sigma$ are as in Theorem 2.4. For all $m \in[0,1)$,

$$
\left\langle\pi_{n}^{0},|x|^{m}\right\rangle \rightarrow\left\langle\pi,|x|^{m}\right\rangle \text {, as } n \rightarrow \infty \text {. }
$$

Suppose additionally that (A8.p) holds for some $p \in(2, \infty)$. Then $(3.1)$ holds for all $m \in[0, p-1)$.

The main step in proving Theorem 3.1 is the following tightness result.

Theorem 3.2. $\sup _{n \in \mathbb{N}}\left\langle\pi_{n},|x|\right\rangle<\infty$, consequently the sequence $\left\{\pi_{n}\right\}_{n \in \mathbb{N}}$ is a tight family of probability measures on $\mathbb{X}$. If in addition (A8.p) holds for some $p \in(2, \infty)$, then $\sup _{n \in \mathbb{N}}\left\langle\pi_{n},|x|^{p-1}\right\rangle<\infty$.

Theorem 3.1 follows from Theorem 3.2 using standard arguments. Indeed, from Theorem 3.2 we have that every subsequence of $\left\{\pi_{n}\right\}$ admits a convergent subsequence. Denoting a typical limit point by $\tilde{\pi}$ we see from Theorem 2.4 that the process $\left(\widehat{Q}^{n}, \widehat{W}^{n}, M_{n}^{-1} \widehat{I}^{n}\right)$, with $\widehat{X}^{n}(0)$ distributed as $\pi_{n}$, converges in distribution to $(Z, B, \xi)$ as in Definition 2.3 with $\mu_{0}=\tilde{\pi}^{0}$, and $b, \Sigma$ as in Theorem 2.4, where $\tilde{\pi}^{0}(A)=\tilde{\pi}\left(A \times \mathbb{R}_{+}^{|\mathcal{E}|} \times \mathbb{R}_{+}^{K}\right), A \in \mathcal{B}\left(\mathbb{R}_{+}^{K}\right)$. Stationarity of $\widehat{Q}^{n}$ implies that $\tilde{\pi}^{0}$ is an invariant measure for the $(b, \Sigma)$-RBM. Theorem 2.6 then gives that $\tilde{\pi}^{0}=\pi$. Thus Theorem 3.1 follows.

Rest of the section is devoted to the proof of Theorem 3.2. We begin with the following moment stability estimate on $\widehat{X}_{x}^{n}$ that is uniform in $n$. 
Theorem 3.3. Suppose that (A8.p) holds for some $p \in[2, \infty)$. Then, there exists a $t_{0} \in(0, \infty)$ such that for all $t \geq t_{0}$,

$$
\lim _{|x| \rightarrow \infty} \sup _{n} \frac{1}{|x|^{p}} \mathbb{E}\left(\left|\widehat{X}_{x}^{n}(t|x|)\right|^{p}\right)=0 .
$$

Proof. We first show that for some $t_{0} \in(0, \infty)$ and all $t \geq t_{0}$,

$$
\lim _{|x| \rightarrow \infty} \sup _{n} \frac{1}{|x|^{p}} \mathbb{E}\left(\left|\widehat{Q}_{x}^{n}(t|x|)\right|^{p}\right)=0 .
$$

Fix $x=(q, u, v) \in \mathbb{X}$. Recall that $Q^{n}$ is given by the representation (2.7) and (2.12) with all processes there written with a superscript $n$. We will now write a slightly modified dynamical description for $\widetilde{Q}^{n}$ that makes explicit the dependence on initial residual times $(u, v)$. We set $u_{i}=\infty$ for $i \notin \mathcal{E}$. We suppress $x$ from the notation unless needed and rewrite $\widetilde{Q}_{i}^{n}$ as

$$
\begin{aligned}
\widetilde{Q}_{i}^{n}(t)= & q+\left(A_{i}(t)-\alpha_{i}\left(t-\sqrt{n} u_{i}\right)^{+}\right)-\left(E_{i}\left(T_{i}(t)\right)-\mu_{i}\left(T_{i}(t)-\sqrt{n} v_{i}\right)^{+}\right) \\
& +\sum_{j \in \mathbb{K}} p_{j i}\left(E_{j}\left(T_{j}(t)\right)-\mu_{j}\left(T_{j}(t)-\sqrt{n} v_{j}\right)^{+}\right)+\sum_{j \in \mathbb{K}}\left(R_{i}^{j}\left(E_{j}\left(T_{j}(t)\right)\right)-p_{j i} E_{j}\left(T_{j}(t)\right)\right) \\
& +\left[\alpha_{i}\left(t-\sqrt{n} u_{i}\right)^{+}+\sum_{j \in \mathbb{K}} p_{j i} \mu_{j}\left(t-\sqrt{n} v_{j}\right)^{+}-\mu_{i}\left(t-\sqrt{n} v_{i}\right)^{+}\right] \\
& +\sum_{j \in \mathbb{K}} p_{j i} \mu_{j}\left(\left(T_{j}(t)-\sqrt{n} v_{j}\right)^{+}-T_{j}(t)\right)+\sum_{j \in \mathbb{K}} p_{j i} \mu_{j}\left(t-\left(t-\sqrt{n} v_{j}\right)^{+}\right) \\
& -\mu_{i}\left[\left(t-\left(t-\sqrt{n} v_{i}\right)^{+}\right)-\left(T_{i}(t)-\left(T_{i}(t)-\sqrt{n} v_{i}\right)^{+}\right)\right] .
\end{aligned}
$$

Thus $\widehat{Q}_{x}^{n}(t)=\Gamma\left(q+N^{n}+b^{n}\right)(t)$, where $N^{n}(t)=N_{1}^{n}(t)+N_{2}^{n}(t)+N_{3}^{n}(t)$ and for $i \in \mathbb{K}$,

$$
\begin{aligned}
N_{1, i}^{n}(t) \doteq & \frac{1}{\sqrt{n}}\left(A_{i}(n t)-\alpha_{i}^{n}\left(n t-\sqrt{n} u_{i}\right)^{+}\right), \\
N_{2, i}^{n}(t) \doteq & \frac{1}{\sqrt{n}}\left[\sum_{j \in \mathbb{K}} p_{j i}\left(E_{j}\left(T_{j}(n t)\right)-\mu_{j}^{n}\left(T_{j}(n t)-\sqrt{n} v_{j}\right)^{+}\right)-\left(E_{i}\left(T_{i}(n t)\right)-\mu_{i}^{n}\left(T_{i}(n t)-\sqrt{n} v_{i}\right)^{+}\right)\right], \\
N_{3, i}^{n}(t) \doteq & \frac{1}{\sqrt{n}} \sum_{j \in \mathbb{K}}\left(R_{i}^{j}\left(E_{j}\left(T_{j}(n t)\right)-p_{j i} E_{j}\left(T_{j}(n t)\right)\right),\right. \\
b_{i}^{n}(t) \doteq & \sqrt{n}\left[\alpha_{i}^{n}\left(t-u_{i} / \sqrt{n}\right)^{+}+\sum_{j \in \mathbb{K}} p_{j i} \mu_{j}^{n}\left(t-v_{j} / \sqrt{n}\right)^{+}-\mu_{i}^{n}\left(t-v_{i} / \sqrt{n}\right)^{+}\right] \\
& +\frac{1}{\sqrt{n}}\left[\sum_{j \in \mathbb{K}} p_{j i} \mu_{j}^{n}\left(\left(T_{j}(n t)-\sqrt{n} v_{j}\right)^{+}-T_{j}(n t)\right)+\sum_{j \in \mathbb{K}} p_{j i} \mu_{j}^{n}\left(n t-\left(n t-\sqrt{n} v_{j}\right)^{+}\right)\right. \\
& \left.-\mu_{i}^{n}\left[\left(n t-\left(n t-\sqrt{n} v_{i}\right)^{+}\right)-\left(T_{i}(n t)-\left(T_{i}(n t)-\sqrt{n} v_{i}\right)^{+}\right)\right]\right] .
\end{aligned}
$$

Define

$$
Z_{x}^{n}(t) \doteq \Gamma\left(q+b^{n}\right)(t), \quad t \geq 0
$$


Using the Lipschitz property of the Skorohod map (see Proposition 2.2), we have

$$
\left|\widehat{Q}_{x}^{n}(t)-Z_{x}^{n}(t)\right| \leq R \sup _{0 \leq s \leq t}\left|N^{n}(s)\right| .
$$

Let $\bar{t} \doteq|x|$ and set $\bar{z}^{n} \doteq Z_{x}^{n}(\bar{t})$. Observing that $\bar{t} \geq \max _{i \in \mathbb{K}}\left\{\frac{u_{i}}{\sqrt{n}}, \frac{v_{i}}{\sqrt{n}}\right\}$ and $T_{i}(t)=t$ for $t \in\left[0, \sqrt{n} v_{i}\right]$, $i \in \mathbb{K}, n \geq 1$, we get

$$
Z_{x}^{n}(t)=\Gamma\left(\bar{z}^{n}+\bar{b}^{n} \iota\right)(t-\bar{t}), \quad t \geq \bar{t},
$$

where $\iota:[0, \infty) \rightarrow[0, \infty)$ is the identity map and $\bar{b}^{n} \doteq \sqrt{n}\left[\alpha^{n}-\left(\mathbb{I}-\mathcal{P}^{\prime}\right) \mu^{n}\right]=\sqrt{n}\left[\left(\mathbb{I}-\mathcal{P}^{\prime}\right) \tilde{\beta}^{n}-\tilde{v}^{n}\right]$.

Next note that

$$
\left|\bar{z}^{n}\right|=\left|\Gamma\left(q+b^{n}\right)(|x|)\right| \leq R\left[|q|+\sup _{0 \leq t \leq|x|}\left|b^{n}(t)\right|\right]
$$

Define $c_{0} \doteq \sup _{i, n}\left\{\alpha_{i}^{n}+3\left(\sum_{j \in \mathbb{K}} p_{j i} \mu_{j}^{n}+\mu_{i}^{n}\right)\right\}$. If $t<\frac{|x|}{\sqrt{n}}$ we see that $\left|b_{i}^{n}(t)\right| \leq c_{0}|x|$ for all $n \geq 1$ and $i \in \mathbb{K}$. On the other hand, if $t \geq \frac{|x|}{\sqrt{n}}$

$$
\left|b^{n}(t)\right| \leq\left|\sqrt{n}\left[\alpha^{n}-\left(\mathbb{I}-\mathcal{P}^{\prime}\right) \mu^{n}\right]\right| t+K c_{0}|x| .
$$

Combining the above observation with (3.7) and the heavy traffic condition (A5), we see that one can find $L_{0} \in(0, \infty)$ such that

$$
\left|\bar{z}^{n}\right| \leq L_{0}|x|
$$

Next, denoting by $\mathcal{C} \doteq\left\{v \in \mathbb{R}^{K}:\left[\mathbb{I}-\mathcal{P}^{\prime}\right]^{-1} v \leq 0\right\}$, we see that from (A5) and (A6) that there exists a $\delta>0$ such that

$$
\inf _{n} \operatorname{dist}\left(\bar{b}_{n}, \partial \mathcal{C}\right) \geq \delta \text {. }
$$

Let $\mathcal{C}_{\delta} \doteq\{v \in \mathcal{C}: \operatorname{dist}(v, \partial \mathcal{C}) \geq \delta\}$ and for $q_{0} \in \mathbb{R}_{+}^{K}$, let $\mathcal{A}\left(q_{0}\right)$ be the collection of all trajectories $\psi:[0, \infty) \rightarrow \mathbb{R}_{+}^{K}$ of the form

$$
\psi(t) \doteq \Gamma\left(q_{0}+v \iota\right)(t), \quad t \geq 0,
$$

where $v$ ranges over all of $\mathcal{C}_{\delta}$. Define the "hitting time to the origin" function

$$
T\left(q_{0}\right) \doteq \sup _{\psi \in \mathcal{A}\left(q_{0}\right)} \inf \{t \in[0, \infty): \psi(t)=0\} .
$$

Lemma 3.1 of [1] shows that

$$
T\left(q_{0}\right) \leq \frac{4 R^{2}}{\delta}\left|q_{0}\right|, \text { and for all } \psi \in \mathcal{A}\left(q_{0}\right), \psi(t)=0 \text { for all } t \geq T\left(q_{0}\right) .
$$

Combining this with (3.6), (3.9) and (3.8) we now have that $Z_{x}^{n}(t)=0$, for all $t \geq L|x|$, where $L \doteq\left[1+\frac{4 R^{2}}{\delta} L_{0}\right]$. Using this in (3.5) we now see that

$$
\left|\widehat{Q}_{x}^{n}(t|x|)\right| \leq R \sup _{0 \leq s \leq t|x|}\left|N^{n}(s)\right|,
$$


for all $t \geq L$ and for all initial conditions $x$. Next we obtain an estimate on the $p^{\text {th }}$ moment of the right side of (3.12). Note that

$$
A_{i}^{n}(t)=A_{i, 0}^{n}\left(\left(t-\sqrt{n} u_{i}\right)^{+}\right)+1_{\left[\sqrt{n} u_{i}, \infty\right)}(t), \quad E_{i}^{n}(t)=E_{i, 0}^{n}\left(\left(t-\sqrt{n} v_{i}\right)^{+}\right)+1_{\left[\sqrt{n} v_{i}, \infty\right)}(t) .
$$

From (A8.p), for some $\kappa_{0} \in(0, \infty)$ (depending on $p$ ) and all $n \geq 1$

$\frac{1}{n^{p / 2}} \mathbb{E} \sup _{0 \leq s \leq t}\left(\left|A_{i, 0}^{n}(n s)-n \alpha_{i}^{n} s\right|^{p}+\left|E_{i, 0}^{n}(n s)-n \mu_{i}^{n} s\right|^{p}+\left|R_{i}^{j}\left(E_{j, 0}^{n}(n s)\right)-p_{j i} E_{j, 0}^{n}(n s)\right|^{p}\right) \leq \kappa_{0}(1+t)$,

for all $i \in \mathcal{E}, j \in \mathbb{K}$. Using these estimates in (3.3), recalling (3.13), and noting that for all $t \geq 0$, $\left[T_{i}(n t)-\sqrt{n} v_{i}\right]^{+} \leq n t$, we obtain for some $\kappa_{1} \in(0, \infty)$,

$$
\mathbb{E} \sup _{0 \leq s \leq t}\left[\left|N_{1, i}^{n}(s)\right|^{p}+\left|N_{2, i}^{n}(s)\right|^{p}+\left|N_{3, i}^{n}(s)\right|^{p}\right] \leq \kappa_{1}\left(1+t^{p / 2}\right) .
$$

Applying this estimate in (3.12) we now have that for all $t \geq L, x \in \mathbb{X}$ and for some $\kappa_{2} \in(0, \infty)$,

$$
\mathbb{E}\left|\widehat{Q}_{x}^{n}(t|x|)\right|^{p} \leq \kappa_{2}\left(1+t^{p / 2}|x|^{p / 2}\right) .
$$

Choosing $t_{0}=L$ the result (3.2) now follows.

It remains to show that for all $t \geq t_{0}$

$$
\lim _{|x| \rightarrow \infty} \sup _{n} \frac{1}{|x|^{p}} \mathbb{E}\left(\left|\widehat{U}_{x}^{n}(t|x|)\right|^{p}\right)=0, \quad \lim _{|x| \rightarrow \infty} \sup _{n} \frac{1}{|x|^{p}} \mathbb{E}\left(\left|\widehat{V}_{x}^{n}(t|x|)\right|^{p}\right)=0 .
$$

Recall $\widehat{V}_{i}^{n}(t|x|)=\frac{1}{\sqrt{n}} V_{i}^{n}(n t|x|)$ and thus

$$
\left|\widehat{V}_{i}^{n}(t|x|)\right|^{p} \leq \frac{1}{n^{p / 2}}\left|\Delta_{i}^{n}\left(E_{i}^{n}\left(T_{i}^{n}(n t|x|)\right)\right)\right|^{p} \leq \frac{1}{n^{p / 2}} \sum_{k=1}^{E_{i}^{n}\left(T_{i}^{n}(n t|x|)\right)}\left|\Delta_{i}^{n}(k)\right|^{p} \leq \frac{1}{n^{p / 2}} \sum_{k=1}^{E_{i, 0}^{n}(n t|x|)}\left|\Delta_{i}^{n}(k)\right|^{p},
$$

where the second inequality above uses the fact that $E_{i}^{n}\left(T_{i}^{n}(n t|x|)\right) \geq 1$ since $V_{i, 0}^{n}=\sqrt{n} v_{i} \leq n t_{0}|x|$ and the last inequality follows on $T_{i}^{n}(t) \leq t$ for all $t \geq 0$.

Using Wald's identity we have for some $\kappa_{3} \in(0, \infty)$, and all $x \in \mathbb{X}, t \geq t_{0}$

$$
\mathbb{E}\left(\left|\widehat{V}_{i}^{n}(t|x|)\right|^{p}\right) \leq \frac{1}{n^{p / 2}} \mathbb{E}\left(E_{i, 0}^{n}(n t|x|)+1\right) \mathbb{E}\left[\Delta_{i}^{n}(1)\right]^{p} \leq \kappa_{3}(1+t|x|) .
$$

This proves the second statement in (3.16). The first statement is shown similarly.

Remark 3.1. Proof of Theorem 3.3 makes use of the Lipschitz property of the Skorohod map. There is a rich collection of multiclass queuing networks for which the corresponding diffusion limit is not given in terms of such a well behaved Skorohod map. Proofs in the current paper (or of [5]) cannot be easily extended to cover such settings.

For $\bar{\delta} \in(0, \infty)$, define the return time to a compact set $C \subset \mathbb{X}$ by $\tau_{C}^{n}(\bar{\delta}) \doteq \inf \left\{t \geq \bar{\delta}: \widehat{X}^{n}(t) \in C\right\}$. The proof of the following result is adapted from that of Proposition 5.3 of [4]. Estimates similar to (3.18), for each fixed $n$, were established in [4] and then used to obtain bounds for the moments of the stationary distribution $\pi_{n}$ for fixed $n$. The key idea in the current work is to use the uniform in $n$ estimate in (3.18) to obtain moment bounds for $\pi_{n}$ that are uniform in $n$. 
Theorem 3.4. Suppose that (A8.p) holds for some $p \in[2, \infty)$. Then, for some constants $c, \bar{\delta} \in$ $(0, \infty)$, and a compact set $C \subset \mathbb{X}$,

$$
\sup _{n} \mathbb{E}\left[\int_{0}^{\tau_{C}^{n}(\bar{\delta})}\left(1+\left|\widehat{X}_{x}^{n}(t)\right|^{p-1}\right) d t\right] \leq c\left(1+|x|^{p}\right), \quad x \in \mathbb{X} .
$$

Proof. By Theorem 3.3, there exists a $\bar{L} \in(0, \infty)$ such that with $C \doteq\{x \in \mathbb{X}:|x| \leq \bar{L}\}$,

$$
\sup _{n} \mathbb{E}\left|\widehat{X}_{x}^{n}\left(t_{0}|x|\right)\right|^{p} \leq \frac{1}{2}|x|^{p}, \quad \forall x \in C^{c},
$$

where $t_{0}$ is as in Theorem 3.3. Let $\bar{\delta} \doteq t_{0} \bar{L}$ and set $\tau_{C}^{n}(\bar{\delta}) \equiv \tau^{n} \doteq \inf \left\{t \geq \bar{\delta}:\left|\widehat{X}_{x}^{n}(t)\right| \leq \bar{L}\right\}$. Define a sequence of stopping times $\sigma_{m}$ as

$$
\sigma_{0} \doteq 0, \quad \sigma_{m}=\sigma_{m-1}+t_{0}\left[\left|\widehat{X}_{x}^{n}\left(\sigma_{m-1}\right)\right| \vee \bar{L}\right], \quad m \in \mathbb{N} .
$$

Note that the dependence of these stopping times on $n$ has been suppressed in notation. Also, let $m_{0}^{n} \doteq \min \left\{m \geq 1:\left|\widehat{X}_{x}^{n}\left(\sigma_{m}\right)\right| \leq \bar{L}\right\}$. Define $\widehat{V}_{n}(x) \doteq \mathbb{E}\left[\int_{0}^{\tau^{n}}\left(1+\left|\widehat{X}_{x}^{n}(t)\right|^{p-1}\right) d t\right], x \in \mathbb{X}$. Then

$$
\widehat{V}_{n}(x) \leq \mathbb{E}\left[\int_{0}^{\sigma_{m_{0}^{n}}}\left(1+\left|\widehat{X}_{x}^{n}(t)\right|^{p-1}\right) d t\right]=\sum_{k=0}^{\infty} \mathbb{E}\left[\int_{\sigma_{k}}^{\sigma_{k+1}}\left(1+\left|\widehat{X}_{x}^{n}(t)\right|^{p-1}\right) d t 1_{k<m_{0}^{n}}\right] .
$$

Let $\mathcal{F}_{t} \doteq \sigma\left\{\widehat{X}_{x}^{n}(s): 0 \leq s \leq t\right\}$ (we suppress $n$ and $x$ in the notation). We claim that for some constant $c_{0} \in(0, \infty)$, and for all $n, k \in \mathbb{N}, x \in \mathbb{X}$,

$$
\mathbb{E}\left[\int_{\sigma_{k}}^{\sigma_{k+1}}\left(1+\left|\widehat{X}_{x}^{n}(t)\right|^{p-1}\right) d t \mid \mathcal{F}_{\sigma_{k}}\right] 1_{k<m_{0}^{n}} \leq c_{0}\left(1+\left|\widehat{X}_{x}^{n}\left(\sigma_{k}\right)\right|^{p}\right) 1_{k<m_{0}^{n}}
$$

The claim is proved below (3.24). Assuming that the claim holds and using this estimate in (3.20) we get by suitable conditioning

$$
\sup _{n} \widehat{V}_{n}(x) \leq c_{0} \sup _{n} \mathbb{E}\left[\sum_{k=0}^{m_{0}^{n}-1}\left(1+\left|\widehat{X}_{x}^{n}\left(\sigma_{k}\right)\right|^{p}\right)\right] .
$$

Next note that $\left\{\widehat{X}_{x}^{n}\left(\sigma_{k}\right)\right\}_{k \geq 1}$ is a Markov chain with the one step transition kernel

$$
\breve{P}_{n}(x, A) \doteq P_{n}^{t_{0}(|x| \vee \bar{L})}(x, A), \quad x \in \mathbb{X}, \quad A \in \mathcal{B}(\mathbb{X}),
$$

where $P_{n}^{t}$ is the transition probability kernel for the Markov process $\widehat{X}_{n}$. Using $(3.15),(3.17)$ and (3.19) one has for some constant $\tilde{b} \in(1, \infty)$,

$$
\sup _{n} \int_{\mathbb{X}} \breve{P}_{n}(x, d y)|y|^{p} \leq|x|^{p}-\frac{1}{2}|x|^{p}+\tilde{b} 1_{[0, \bar{L}]}(|x|) .
$$

Using Theorem 14.2.2 of [10] we have that

$$
\sup _{n} \mathbb{E} \sum_{k=0}^{m_{0}^{n}-1}\left[1+\left|\widehat{X}_{x}^{n}\left(\sigma_{k}\right)\right|^{p}\right] \leq 3\left[|x|^{p}+2 \sup _{n} \mathbb{E} \sum_{k=0}^{m_{0}^{n}-1} \tilde{b} 1_{C}\left(\widehat{X}_{x}^{n}\left(\sigma_{k}\right)\right)\right]=3\left[|x|^{p}+2 \tilde{b} 1_{[0, \bar{L}]}(|x|)\right]
$$


where the second equality follows from the fact that whenever $1 \leq k \leq m_{0}^{n}-1,\left|\widehat{X}_{x}^{n}\left(\sigma_{k}\right)\right|>\bar{L}$ (we assume without loss of generality $\bar{L}>2$ ). The inequality (3.18) now follows on using the above estimate in (3.22).

Thus it only remains to prove the claim in (3.21). By an application of strong Markov property this is equivalent to showing for some $c_{0} \in(0, \infty)$ and all $n, x$

$$
\mathbb{E}\left[\int_{0}^{\sigma_{1}}\left(1+\left|\widehat{X}_{x}^{n}(t)\right|^{p-1}\right) d t\right] \leq c_{0}\left(1+|x|^{p}\right)
$$

Since $\left|\widehat{X}_{x}^{n}(t)\right| \doteq\left|\widehat{Q}_{x}^{n}(t)\right|+\left|\widehat{U}_{x}^{n}(t)\right|+\left|\widehat{V}_{x}^{n}(t)\right|$, let us first consider $\mathbb{E}\left[\int_{0}^{\sigma_{1}}\left(1+\left|\widehat{Q}_{x}^{n}(t)\right|^{p-1}\right) d t\right]$. From definition of $\sigma_{1}$, we see that

$$
\sigma_{1} \leq c_{1}(1+|x|)
$$

for some constant $c_{1} \in(0, \infty)$. Note that

$$
\begin{aligned}
\sup _{n} \sup _{0 \leq t \leq \sigma_{1}} \mathbb{E}\left|\widehat{Q}_{x}^{n}(t)\right|^{p-1} & =\sup _{n} \sup _{0 \leq t \leq \sigma_{1}} \mathbb{E}\left|\Gamma\left(q+N^{n}+b_{n}\right)(t)-Z_{x}^{n}(t)+Z_{x}^{n}(t)\right|^{p-1} \\
& \leq \sup _{n} R \mathbb{E} \sup _{0 \leq t \leq \sigma_{1}}\left|N^{n}(t)\right|^{p-1}+\sup _{n} \sup _{0 \leq t \leq \sigma_{1}} \mathbb{E}\left|Z_{x}^{n}(t)\right|^{p-1} .
\end{aligned}
$$

Following steps analogous to those leading to $(3.8)$ we see that for some $c_{2} \in(0, \infty)$

$$
\sup _{0 \leq t \leq \sigma_{1}} \mathbb{E}\left|Z_{x}^{n}(t)\right|^{p-1} \leq c_{2}\left(1+|x|^{p-1}\right), \quad \forall x \in \mathbb{X} .
$$

Using this estimate along with (3.14) and (3.26) in (3.27) we now have for some $c_{3} \in(0, \infty)$ and $x \in \mathbb{X}$

$$
\mathbb{E}\left[\int_{0}^{\sigma_{1}}\left(1+\left|\widehat{Q}_{x}^{n}(t)\right|^{p-1}\right) d t\right] \leq c_{3}\left(1+|x|^{p}\right) .
$$

Noting that $\left|\widehat{U}_{i}^{n}(t)\right|^{p-1} \leq|x|^{p-1}+\frac{1}{n^{(p-1) / 2}} \sum_{k=1}^{A_{i}^{n}(n t)}\left|\eta_{i}^{n}(k)\right|^{p-1}$, an argument similar to that leading to (3.17) shows that for some $c_{4} \in(0, \infty)$, and all $t \geq 0, x \in \mathbb{X}, \mathbb{E}\left|\widehat{U}_{x}^{n}(t)\right|^{p-1} \leq c_{4}\left[1+|x|^{p-1}+t\right]$. Thus, for some $c_{5} \in(0, \infty)$,

$$
\mathbb{E} \int_{0}^{\sigma_{1}}\left|\widehat{U}_{x}^{n}(t)\right|^{p-1} d t \leq c_{5}\left[1+|x|^{p}\right]
$$

In a similar manner, one sees for some $c_{6} \in(0, \infty), \mathbb{E} \int_{0}^{\sigma_{1}}\left|\widehat{V}_{x}^{n}(t)\right|^{p-1} d t \leq c_{6}\left[1+|x|^{p}\right]$. The estimate in (3.25) now follows on combining the above inequalities with (3.28) and (3.26). This proves (3.21) and the result follows.

Theorem 3.5. Let $f: \mathbb{X} \rightarrow \mathbb{R}_{+}$, and define for $\bar{\delta} \in(0, \infty)$, and a compact set $C \subset \mathbb{X}$

$$
V_{n}(x) \doteq \mathbb{E}\left[\int_{0}^{\tau_{C}^{n}(\bar{\delta})} f\left(\widehat{X}_{x}^{n}(t)\right) d t\right], \quad x \in \mathbb{X} .
$$

If $\sup _{n} V_{n}$ is everywhere finite and uniformly bounded on $C$, then there exists $\bar{\kappa} \in(0, \infty)$ such that for all $n \in \mathbb{N}, t>0, x \in \mathbb{X}$

$$
\frac{1}{t} \mathbb{E}\left[V_{n}\left(\widehat{X}_{x}^{n}(t)\right)\right]+\frac{1}{t} \int_{0}^{t} \mathbb{E}\left[f\left(\widehat{X}_{x}^{n}(s)\right)\right] d s \leq \frac{1}{t} V_{n}(x)+\bar{\kappa} .
$$


The proof of Theorem 3.5 follows from that of Proposition 5.4 in [4]. For the sake of completeness we provide a sketch in the Appendix.

Proof of Theorem 3.2. Suppose that (A8.p) holds for some $p \in[2, \infty)$. We apply Theorem 3.5 with $f(x) \doteq 1+|x|^{p-1}$ for $x \in \mathbb{X}$ and $\bar{\delta}, C$ as in Theorem 3.4. To prove the result it suffices to show that for all $n \in \mathbb{N},\left\langle\pi_{n}, f\right\rangle \doteq \int_{\mathbb{X}} f(x) \pi_{n}(d x) \leq \bar{\kappa}$. Since $\pi_{n}$ is an invariant measure, for any non-negative, real measurable function $\Phi$ on $\mathbb{X}$,

$$
\int_{\mathbb{X}} \mathbb{E}\left[\Phi\left(\widehat{X}_{x}^{n}(t)\right)\right] \pi_{n}(d x)=\left\langle\pi_{n}, \Phi\right\rangle
$$

Fix $k \in \mathbb{N}$ and let $V_{n}^{k}(x) \doteq V_{n}(x) \wedge k$. Let

$$
\Psi_{n}^{k}(x) \doteq \frac{1}{t} V_{n}^{k}(x)-\frac{1}{t} \mathbb{E}\left[V_{n}^{k}\left(\widehat{X}_{x}^{n}(t)\right)\right]
$$

From (3.30), we have that $\int_{\mathbb{X}} \Psi_{n}^{k}(x) \pi_{n}(d x)=0$. Let $\Psi_{n}(x) \doteq \frac{1}{t} V_{n}(x)-\frac{1}{t} \mathbb{E}\left[V_{n}\left(\widehat{X}_{x}^{n}(t)\right)\right]$. Monotone convergence theorem yields that $\Psi_{n}^{k}(x) \rightarrow \Psi_{n}(x)$ as $k \rightarrow \infty$. Next we will show that $\Psi_{n}^{k}(x)$ is bounded from below for all $x \in \mathbb{X}$. If $V_{n}(x) \leq k$,

$$
\Psi_{n}^{k}(x)=\frac{1}{t} V_{n}^{k}(x)-\frac{1}{t} \mathbb{E}\left[V_{n}^{k}\left(\widehat{X}_{x}^{n}(t)\right)\right] \geq \frac{1}{t} V_{n}(x)-\frac{1}{t} \mathbb{E}\left[V_{n}\left(\widehat{X}_{x}^{n}(t)\right)\right] \geq-\bar{\kappa},
$$

where the last inequality follows from (3.29). On the other hand, if $V_{n}(x) \geq k$

$$
\Psi_{n}^{k}(x)=\frac{1}{t} k-\frac{1}{t} \mathbb{E}\left[V_{n}^{k}\left(\widehat{X}_{x}^{n}(t)\right)\right] \geq 0,
$$

where the second inequality follows on noting that $V_{n}^{k} \leq k$. Hence $\Psi_{n}^{k}(x) \geq-\bar{\kappa}$ for all $x \in \mathbb{X}$. By an application of Fatou's Lemma we conclude that

$$
\int_{\mathbb{X}} \Psi_{n}(x) \pi_{n}(d x) \leq \liminf _{k \rightarrow \infty} \int_{\mathbb{X}} \Psi_{n}^{k}(x) \pi_{n}(d x)=0 .
$$

From (3.29), $\Psi_{n}(x) \geq \frac{1}{t} \int_{0}^{t} \mathbb{E}\left[f\left(\widehat{X}_{x}^{n}(s)\right)\right] d s-\bar{\kappa}$. Combining this with (3.32) we have

$$
0 \geq \int_{\mathbb{X}} \Psi_{n}(x) \pi_{n}(d x) \geq \frac{1}{t} \int_{0}^{t} \int_{\mathbb{X}} \mathbb{E}\left[f\left(\widehat{X}_{x}^{n}(s)\right)\right] \pi_{n}(d x) d s-\bar{\kappa} .
$$

Using (3.30) once more we now have that $\left\langle\pi_{n}, f\right\rangle \leq \bar{\kappa}$. This completes the proof.

\section{Appendix}

Proof of Theorem 3.5. The proof is adapted from Proposition 5.4 of [4]. We begin by showing that:

$$
\text { For all } m \in \mathbb{N}, \quad \mathbb{E}\left[\int_{0}^{\tau_{C}^{n}(m \bar{\delta})} f\left(\widehat{X}_{x}^{n}(t)\right) d t\right] \leq V_{n}(x)+b_{1} m \bar{\delta}, \quad x \in \mathbb{X}
$$


where $b_{1} \doteq \sup _{n} \sup _{x \in C} V_{n}(x) / \bar{\delta}$. The proof is by induction. For $m=1$, the inequality in $(\mathbb{A} 1)$ holds trivially. Suppose now that $(\mathbb{A} 1)$ holds for $m=k \in \mathbb{N}$. In what follows, instead of indicating the dependence on the initial condition as a subscript to $\widehat{X}^{n}$, we will indicate it in the expectation operation. For example, $\mathbb{E}\left[f\left(\widehat{X}_{x}^{n}(t)\right)\right]$ will be written as $\mathbb{E}_{x}\left[f\left(\widehat{X}^{n}(t)\right)\right]$, etc. Using the strong Markov property of $\widehat{X}^{n}$ we have that

$$
\begin{aligned}
\mathbb{E}_{x}\left[\int_{0}^{\tau_{C}^{n}((k+1) \bar{\delta})} f\left(\widehat{X}^{n}(t)\right) d t\right] & \leq \mathbb{E}_{x}\left[\int_{0}^{\tau_{C}^{n}(\bar{\delta})} f\left(\widehat{X}^{n}(t)\right) d t\right]+\mathbb{E}_{x}\left[\mathbb{E}_{\widehat{X}_{\tau_{C}^{n}(\bar{\delta})}^{n}}\left[\int_{0}^{\tau_{C}^{n}(k \bar{\delta})} f\left(\widehat{X}^{n}(t)\right) d t\right]\right] \\
& \leq V_{n}(x)+\sup _{x \in C} \mathbb{E}_{x}\left[\int_{0}^{\tau_{C}^{n}(k \bar{\delta})} f\left(\widehat{X}^{n}(t)\right) d t\right] \\
& \leq V_{n}(x)+\sup _{n} \sup _{x \in C} V_{n}(x)+b_{1} k \bar{\delta},
\end{aligned}
$$

where the last inequality follows from the induction hypothesis. Proof of $(\mathbb{A} 1)$ now follows on noting that the right side of $(\mathbb{A} 2)$ coincides with $V_{n}(x)+b_{1}(k+1) \bar{\delta}$. Using the monotonicity in $m$ of the expression on the left side of $(\mathbb{A} 1)$ we now have that for all $t \geq \bar{\delta}$,

$$
\mathbb{E}_{x}\left[\int_{0}^{\tau_{C}^{n}(t)} f\left(\widehat{X}^{n}(s)\right) d s\right] \leq V_{n}(x)+2 b_{1} t .
$$

Note that $(\mathbb{A} 3)$ is trivially satisfied for all $t<\bar{\delta}$. Thus $(\mathbb{A} 3)$ holds for all $t \geq 0$. Using the strong Markov property once again, we have (see proof of Proposition 5.4 of [4] for analogous arguments)

$$
\begin{aligned}
\mathbb{E}_{x}\left[V_{n}\left(\widehat{X}^{n}(t)\right)\right] & \leq V_{n}(x)-\int_{0}^{t} \mathbb{E}_{x}\left[f\left(\widehat{X}^{n}(s)\right)\right] d s+\mathbb{E}_{x}\left[\mathbb{E}_{\widehat{X}_{\tau_{C}^{n}(\bar{\delta})}^{n}}\left[\int_{0}^{\tau_{C}^{n}(t)} f\left(\widehat{X}^{n}(s)\right) d s\right]\right] \\
& \leq V_{n}(x)-\int_{0}^{t} \mathbb{E}_{x}\left[f\left(\widehat{X}^{n}(s)\right) d s\right]+\sup _{n} \sup _{x \in C} \mathbb{E}_{x}\left[\int_{0}^{\tau_{C}^{n}(t)} f\left(\widehat{X}^{n}(s)\right)\right] d s \\
& \leq V_{n}(x)-\int_{0}^{t} \mathbb{E}_{x}\left[f\left(\widehat{X}^{n}(s)\right)\right] d s+\sup _{n} \sup _{x \in C} V_{n}(x)+2 b_{1} t
\end{aligned}
$$

where the last inequality follows from $(\mathbb{A} 3)$. We obtain (3.29) from dividing both sides in $(\mathbb{A} 4)$ by $t$ and putting $\bar{\kappa} \doteq\left(\frac{2}{\delta}+1\right) \sup _{n} \sup _{x \in C} V_{n}(x)$.

Acknowledgment. We thank the associate editor and the anonymous referee for their careful reading and their suggestions.

\section{References}

[1] R. Atar, A. Budhiraja, and P. Dupuis. On positive recurrence of constrained diffusion processes. Annals of Probability, 29(2):979-1000, 2001.

[2] A. Budhiraja and A. Ghosh. Diffusion approximations for controlled stochastic networks: An asymptotic bound for the value function. Ann. Appl. Probab., 16(4):1962-2006, 2006. 
[3] J. G. Dai. On positive Harris recurrence of queueing networks: A unified approach via fluid limit models. Annals of Applied Probability, 5:49-77, 1995.

[4] J. G. Dai and S. P. Meyn. Stability and convergence of moments for multiclass queueing networks via fluid limit models. IEEE Transactions on Automatic Control, 40:1889-1904, 1995 .

[5] D. Gamarnik and A. Zeevi. Validity of heavy traffic steady-state approximations in open queueing networks. Annals of Applied Probability, 16(1):56-90, 2006.

[6] J. M. Harrison and M. I. Reiman. Reflected Brownian motion on an orthant. Ann. Probab., 9(2):302-308, 1981.

[7] J. M. Harrison and R. J. Williams. Brownian models of open queueing networks with homogeneous customer populations. Stochastics, 22(2):77-115, 1987.

[8] J. R. Jackson. Networks of waiting lines. Operations Res., 5:518-521, 1957.

[9] S. P. Meyn and D. Down. Stability of generalized Jackson networks. Ann. of Appl. Probab., 4(1):124-148, 1994.

[10] S. P. Meyn and R. L. Tweedie. Markov Chains and Stochastic Stability. Springer-Verlag, London, 1993.

[11] M. I. Reiman. Open queueing networks in heavy traffic. Mathematics of Operations Research, 9(3):441-458, 1984.

[12] K. Sigman. The stability of open queueing networks. Stochastic Process. Appl., 35(1):11-25, 1990.

[13] Walter L. Smith. On the cumulants of renewal processes. Biometrika, 46(1/2):1-29, 1959. 